\title{
ABSORÇÃO DE CHUMBO E POTENCIAL DE FITORREMEDIAÇÃO DE CANAVALIA ENSIFORMES L. $\left({ }^{1}\right)$
}

\author{
SOLANGE ROMEIRO $\left({ }^{2}\right)$; ANA MARIA MAGALHÃES ANDRADE LAGÔA $\left(\left(^{*}\right)\right.$; \\ PEDRO ROBERTO FURLANI $\left({ }^{3}\right)$; CLEIDE APARECIDA DE ABREU $\left({ }^{3}\right)$; \\ BRUNO FERNANDO FARIA PEREIRA $\left({ }^{3}\right)$
}

\begin{abstract}
RESUMO
A fitoextração é uma importante técnica usada para descontaminar áreas poluidas por metais pesados. Conseqüentemente, o entendimento das respostas fisiológicas de espécies tropicais com relação à tolerância aos níveis crescentes de contaminantes é fundamental considerando seu possível uso para fitoextração de metais pesados de áreas contaminadas. O objetivo deste estudo foi conhecer o potencial fitoextrator de Canavalia ensiformes L. para o chumbo $(\mathrm{Pb})$. As plantas foram cultivadas em solução nutritiva em casa de vegetação sobre condições parcialmente controladas. $\mathrm{O} \mathrm{Pb}$ foi testado nas concentrações de 100, 200 e $400 \mathrm{mmol} . \mathrm{L}^{-1}$. O experimento foi montado em blocos ao acaso, usando um esquema fatorial 4 x 1, com três repetições. Foram realizadas análises fisiológicas com o acompanhamento de sintomas, das massas frescas e secas tanto da parte aérea como das raízes, da área foliar, das alturas das plantas e do conteúdo de $\mathrm{Pb}$ na solução nutritiva, nas raízes e na parte aérea das plantas. As amostras das plantas (parte aérea e raiz) e da solução nutritiva foram submetidas às análises químicas para determinação de $\mathrm{Pb}$ usando a digestão via seca. As plantas de Canavalia ensiformes foram boas acumuladoras e tolerantes ao $\mathrm{Pb}$, demonstrando seu potencial como fitoextratoras para esse metal.
\end{abstract}

Palavras-chave: fitoextração, metal pesado, sistema hidropônico.

\section{LEAD UPTAKE AND POTENTIAL FOR FITOREMEDIATION OF CANAVALIA ENSIFORMES L. (1)}

\begin{abstract}
Phytoextraction is an important technique used for decontamination of areas polluted by heavy metals. Consequently, understanding the physiological responses in relation to the tolerance of tropical species subjected to increasing levels of contamination is fundamental before considering their use as phytoextractans in contaminated areas. The objective of our study was to assess the potential of Canavalia ensiformes L. for the phytoextraction of lead $(\mathrm{Pb})$. Plants were cultivated with nutrient solution in greenhouse under partially controlled conditions. Pb was tested at concentrations of 100, 200 and 400 $\mu \mathrm{mol} . \mathrm{L}^{-1}$. The experimental set-up was a randomized blocks design, using a $4 \times 1$ factorial scheme, with three replicates. Follow-ups of physiological symptoms were carried out, determining fresh and dried mass for shoot and root, leaf area, plant height, $\mathrm{Pb}$ content in nutrient solution and in roots and shoot of the plants. The samples of shoot and roots and of the nutrient solution were submitted to chemical analysis for the determination of $\mathrm{Pb}$, using dry digestion. The Canavalia ensiformes plants proved to be both accumulators and tolerant to $\mathrm{Pb}$, demonstrating their potential as a phytoextractants.
\end{abstract}

Key words: phytoremediation, heavy metal, hydroponic system.

$\left({ }^{1}\right)$ Recebido para publicação em 11 de abril de 2006 e aceito em 7 de dezembro de 2006.

$\left({ }^{2}\right)$ Centro de Pesquisa e Desenvolvimento Ecofisiologia e Biofísica, Instituto Agronômico, Campinas (SP), Brasil. E-mail: alagoa@iac.sp.gov.br $\left(^{*}\right)$ autor correspondente.

$\left({ }^{3}\right)$ Centro de Solos e Recursos Ambientais, Instituto Agronômico, Campinas (SP) - Brasil. 


\section{INTRODUÇÃO}

$\mathrm{Na}$ atualidade, a crescente e devastadora degradação do meio ambiente tem como base o uso intenso e inadequado de fertilizantes e pesticidas no solo que, aliado ao aumento das atividades industriais e de mineração, têm sido apontados como os principais responsáveis pela contaminação do solo, cursos de água e lençol freático por metais pesados (Malavolta, 1994).

Entre os vários poluentes existentes, o chumbo $(\mathrm{Pb})$ é o maior contaminante de solo (GRATÃo et al., 2005) e o maior problema ambiental diante do mundo moderno (SHEN et al., 2002) sendo, notoriamente, o metal pesado que oferece maior risco de envenenamento aos seres humanos, especialmente às crianças (LASAT, 2002).

A Companhia de Tecnologia de Saneamento Ambiental (CETESB) divulgou em maio de 2005 a existência de 1.504 áreas contaminadas no Estado de São Paulo, sendo a maior parte dessas áreas cadastradas referente a postos de abastecimento de combustível. Os números da contaminação ambiental na área industrial, porém, também são expressivos (CETesb, 2005).

Apesar de não ser um elemento essencial, o $\mathrm{Pb}$ é facilmente absorvido e acumulado em diferentes partes das plantas. A absorção de $\mathrm{Pb}$ é regulada pelo $\mathrm{pH}$, tamanho de partículas e capacidade de troca de cátions dos solos (CTC), assim como pela exsudação e outras características físico-químicas. A absorção do solo para as raízes se dá através da membrana plasmática, provavelmente, por canais catiônicos, como os canais de cálcio. As raízes são capazes de acumular quantidades significativas desse metal pesado (MP) e, simultaneamente, restringir sua translocação para a parte aérea (LANE e MARTIN, 1977). Em baixas concentrações, o $\mathrm{Pb}$ move-se na raiz, predominantemente, via apoplasto e através do córtex e acumula-se perto da endoderme. A endoderme age como uma barreira parcial para a translocação do $\mathrm{Pb}$ das raízes para a parte aérea. Essa pode ser uma das razões para o grande acúmulo de $\mathrm{Pb}$ nas raízes em relação à parte aérea (JonEs et al., 1973; Verma e DubEY, 2003). Romeiro (2005), analisando plantas de mamona que foram desenvolvidas em hidroponia com a presença de $\mathrm{Pb}$ na solução nutritiva, verificou um grande acúmulo de $\mathrm{Pb}$ nas raízes, demonstrando um alto nível de absorção e de acúmulo desse metal nesse órgão. Michalska (2001), trabalhando com três cultivares de alface, demonstrou que $0,5 \mathrm{mM}$ de $\mathrm{Pb}$ adicionado à solução nutritiva provocou maiores acúmulos $\mathrm{Pb}$ nas raízes. Em altas concentrações, a parede celular é danificada e grande quantidade do
MP entra nas células. Estudos das ultraestruturas revelaram o $\mathrm{Pb}$ presente, principalmente, nos espaços intercelulares, parede celular e vacúolos, e pouco depósito no retículo endoplasmático, dictiossomas e vesículas derivadas dos dictiossomas. A parede e o vacúolo juntos corresponderam a $96 \%$ do Pb absorvido (Wierzbicka e ANTOSIEWicz, 1993).

Excesso de $\mathrm{Pb}$ causa vários sintomas de toxicidade em plantas, como redução de crescimento, clorose e escurecimento do sistema radicular. A inibição do crescimento radicular parece ser resultado de uma inibição induzida pelo $\mathrm{Pb}$ na divisão celular do meristema da raiz (Eun et al., 2000). $\mathrm{O} \mathrm{Pb}$ inibe a fotossíntese, altera a nutrição mineral e o balanço hídrico, modifica o estado hormonal e afeta a estrutura e permeabilidade da membrana (SHARMA e Dubey, 2005).

A remediação de áreas contaminadas é uma exigência legal e um compromisso social que precisam ser executados, criando demandas tecnológicas, oportunidades de pesquisa científica e possibilidades de negócios. Pode ser conseguido por inúmeras tecnologias que envolvem processos químicos ou físicos que são, em geral, tecnicamente difíceis e de custos elevados. Dentre as inúmeras tecnologias para remediação de solos contaminados, destacam-se a biorremediação e a fitorremediação como opções para promover a destoxificação do local ou a remoção de elementos contaminantes do solo (Accioly e Siqueira, 2000).

Na fitoextração, as plantas agem removendo, armazenando, transferindo, estabilizando e tornando inofensivos os metais pesados presentes no solo. Emprega plantas hiperacumuladoras para remover os metais do solo pela absorção e pelo acúmulo nas raízes e na parte aérea, podendo ser, posteriormente, dispostas em aterros sanitários ou recicladas para a recuperação do metal. Essas plantas são capazes de tolerar, absorver e translocar altos níveis de metais pesados que seriam tóxicos a qualquer outro organismo (KHAN et al., 2000).

A planta ideal para fitoextração de metais pesados deve ser tolerante aos seus altos níveis, acumular grandes quantidades na parte aérea, ter alta taxa de crescimento, produzir muita biomassa e ter sistema radicular abundante (GARBISU e ALKORTA, 2001). Outra maneira de se avaliar o potencial fitoextrator da espécie vegetal é o fator de transferência $(\mathfrak{t})$, definido como a razão entre a concentração total do contaminante na planta e a concentração total do contaminante no solo, proposta por LUBben e SAUERBECK (1991). Valores elevados de $t$ são desejáveis para fitoextração e quanto maior este fator, maior a absorção do contaminante (HENRY, 2000). 
Existem vários trabalhos que analisam o crescimento de diferentes espécies na presença de $\mathrm{Pb}$. Em plantas de beterraba (Beta vulgaris [B. vulgaris var. saccharifera] hybrid Monohil) crescidas em condições hidropônicas na presença de $\mathrm{Pb}$, verificou-se aumento na massa seca das raízes e nenhuma mudança na parte aérea, porém houve aumento na razão raiz: parte aérea (LARBI et al., 2002). Em Brassica albograba crescida na presença de $\mathrm{Pb}$, verificou-se que a maior parte desse metal estava nas raízes. Neste caso, o conteúdo relativo de água nas folhas caiu à medida que aumentou a concentração de $\mathrm{Pb}$ nas raízes (HorNg et al., 2002). O desenvolvimento de Sesbania aculeata não foi modificado pela presença de $\mathrm{Pb}$ e a maior parte desse elemento ficou concentrada nas raízes e bem pouco na parte aérea (RAMANI et al., 2002). Em plantas de Carex rostrata, Eriophorum angustifolium e Phragmites australis, crescidas em condições hidropônicas, não ocorreram modificações no crescimento quando na presença de $\mathrm{Pb}$ e, como em outros casos já descritos, o $\mathrm{Pb}$ absorvido concentrou-se nas raízes (STOLTz e GREGER, 2002). Em Pinus radiata, verificou-se que o $\mathrm{Pb}$ concentrado nas raízes foi observado exclusivamente nas paredes celulares destes órgãos e em Chamaecytsus palmensis na lamela média e nos espaços intercelulares (JARVIS e LEUNG, 2001 e 2002).

Segundo TANG et al. (2001), as espécies Elsholtzia haichowensis e Commelina communis, crescidas em solução hidropônica cujas concentrações variaram de 66 a $224 \mathrm{mg} \mathrm{kg}^{-1}$ de $\mathrm{Pb}$ acumularam $\mathrm{Pb}$ em maior concentração nas raízes que na parte aérea das plantas. BOONYAPOOKANA et al. (2005), estudando o potencial hiperacumulador para o $\mathrm{Pb}$ de girassol, tabaco e vetiver concluíram que as três acumulam $\mathrm{Pb}$ nas folhas e nos caules, sendo a primeira a mais eficiente.

Diante da importância da fitoextração como técnica remediadora para áreas contaminadas por chumbo, criou-se a necessidade de estudos que relacionam a tolerância de espécies de clima tropical, desenvolvidas em níveis crescentes de contaminação, com os aspectos fisiológicos dessas plantas, para que possam ser efetivamente cultivadas como fitoextratoras nas áreas contaminadas no Brasil.

O objetivo deste trabalho foi avaliar o desenvolvimento de Canavalia ensiformes L. (feijão-deporco) na presença de chumbo e seu potencial fitoextrator para esse metal pesado.

\section{MATERIAL E MÉTODOS}

O experimento foi desenvolvido em casa de vegetação em cultivo hidropônico sob telado e estrutura metálica em condições semicontroladas de luz e temperatura, instalado no Centro Experimental
Central do Instituto Agronômico, em Campinas (SP), Brasil, em latitude $22^{\circ} 24^{\prime}$ Sul, longitude $44^{\circ} 05^{\prime}$ Oeste e altitude de 674 metros, no período de 26 de março a 4 de maio de 2004.

Os tratamentos constituiram-se de concentrações crescentes de $\mathrm{Pb}(0,100 ; 200$ e $400 \mu \mathrm{mol}$ $\mathrm{L}^{-1}$ ), em solução nutritiva (FURLANI e FURLANI, 1988), em sistema hidropônico, usando o acetato de chumbo $(\mathrm{Pb}$ $\left.\left(\mathrm{CH}_{3} \mathrm{COO}\right)_{2} \cdot 3 \mathrm{H}_{2} \mathrm{O}\right)$. As plantas utilizadas foram de feijão-de-porco (Canavalia ensiformis L.).

O delineamento experimental adotado foi o de blocos inteiramente casualizados, em esquema fatorial $4 \times 1$, com três repetições, sendo cinco plantas por parcela.

O emprego do sistema hidropônico para analisar o potencial fitoextrator de plantas é extremamente adequado, pois possibilita avaliações de raízes e interações com os nutrientes do substrato, como demonstram as pesquisas realizadas por Qu et al. (2003).

As sementes foram colocadas para germinar em papel tipo CEL-065 umedecido e mantidas em germinadores com temperatura de 20 a $35 \mathrm{C}^{\circ}$, durante seis dias, de acordo com as regras para análise de sementes (BRASIL, 1992).

Após sete dias, as plantas foram transferidas para os vasos, contendo solução nutritiva arejada constantemente com pedras porosas alimentadas por mangueiras ligadas em um motocompressor e mantidas em casa de vegetação. Depois de onze dias, trocou-se a solução nutritiva dos vasos por solução tratada com concentrações crescentes de chumbo. As plântulas foram mantidas por um período de 28 dias em exposição ao metal, monitorando-se o pH da solução em 5,5 por meio de medidas periódicas, adicionado-se $\mathrm{HCl} 1 \mathrm{~N}$, quando necessário.

Após oito dias da aplicação de $\mathrm{Pb}$, três plantas de cada tratamento foram marcadas para a identificação, realizando-se medidas de altura em um intervalo de seis dias, totalizando-se quatro medidas. Essas plantas foram separadas para realização das medidas das áreas foliares por meio do equipamento Model L. I 3.100 - Area MeterSR n. ${ }^{\circ}$ LAM 1018, Licor - USA.

No fim do experimento, as partes aéreas e as raízes foram separadas, lavadas com água deionizada e secas em estufa em temperatura entre 65 e $75^{\circ} \mathrm{C}$ até a massa constante. Em seguida, o material foi triturado em moinho tipo Willey, equipado com peneira de $1 \mathrm{~mm}$, para as análises do conteúdo de $\mathrm{Pb}$. Fez-se a digestão via seca, por incineração das amostras e dissolução das cinzas com $\mathrm{HCl}$ e o $\mathrm{Pb}$ foi determinado por espectrofotometria de chama induzida por plasma (ICP-AES-Jobin Yvon ${ }^{\circledR}$ ) (ABREU et al., 2000). 
$\mathrm{O}$ potencial das plantas em extrair o $\mathrm{Pb}$ do solo foi avaliado pelo coeficiente de fitoextração ou fator de transferência $t$, por meio da equação: $t=\mathrm{Pb}$ total na planta/ $\mathrm{Pb}$ na solução. Esses cálculos foram feitos considerando-se o conteúdo de $\mathrm{Pb}$ na parte aérea somado ao das raízes, em todas as concentrações aplicadas. Quanto maior esse fator, maior a absorção do contaminante (HeNRY, 2000).

Realizou-se a análise de variância (teste F) e o teste de Tukey a $5 \%$ de probabilidade e quando houve significância, foram realizadas análises de regressão polinomial quadrática para comparação entre os níveis de concentrações de $\mathrm{Pb}$.

\section{RESULTADOS E DISCUSSÃO}

Houve diminuição do crescimento em $C$. ensiformes, sendo esta diretamente proporcional ao aumento das concentrações de $\mathrm{Pb}$ aplicadas, com coeficiente de regressão próximo de 1,0 (Figura 1). Essas diferenças foram estatisticamente significativas, a $5 \%$. Esses resultados são semelhantes ao experimento de MARQUES et al. (2000), realizado com espécies arbóreas cultivadas em solo contaminado por vários metais pesados, no qual o crescimento em altura das espécies foi influenciado pela contaminação do solo.

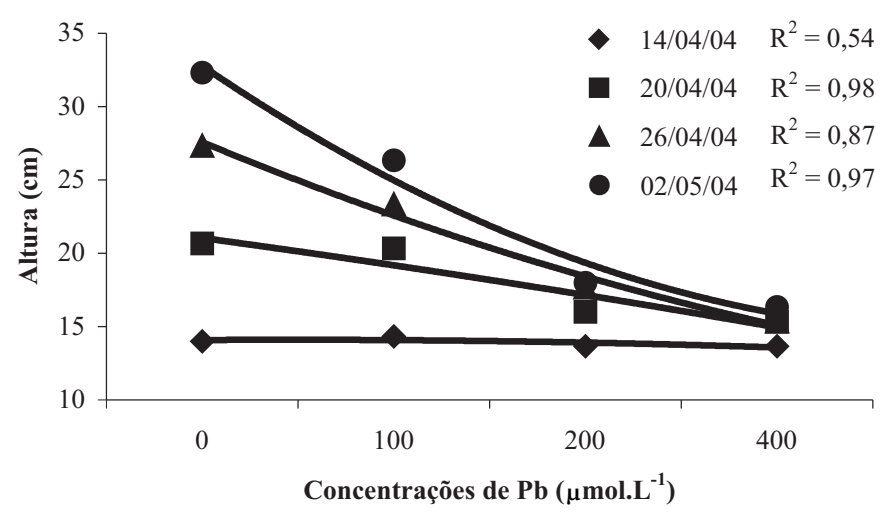

Figura 1. Medidas das alturas de plantas de feijão-de-porco (Canavalia ensiformes L.), cultivadas em sistema hidropônico e submetidas a diferentes concentrações de chumbo. Equações de regressão polinomial quadrática, estatisticamente significativas a $5 \%$, estimadas pelo programa SANEST.

Em relação à área foliar, massa seca da parte aérea e massa seca de raiz de feijão-de-porco houve grande redução até a concentração de $200 \mu \mathrm{mol} . \mathrm{L}^{-1}$ de $\mathrm{Pb}$; a partir daí, o aumento da concentração do metal não alterou mais essas medidas. A regressão polinomial evidencia um coeficiente de determinação alto, demonstrando que com o aumento das concentrações aplicadas de $\mathrm{Pb}$, houve diminuição desses atributos (Figuras 2; 3-A e 3-B). Segundo KosobruKHov et al. (2004), a aplicação de $\mathrm{Pb}$ provoca considerável decréscimo na massa seca de partes de plantas. Em Vetiver zizanioides e $V$. nemoralis a biomassa das plantas diminuiu com o aumento da concentração de $\mathrm{Pb}$ aplicada (CHANTACHON et al., 2004). No entanto, apesar dessas diferenças, as plantas tratadas com 100 e $200 \mathrm{mmol} . \mathrm{L}^{-1}$ ainda ficaram vigorosas (Figuras 4-A e 4-B).

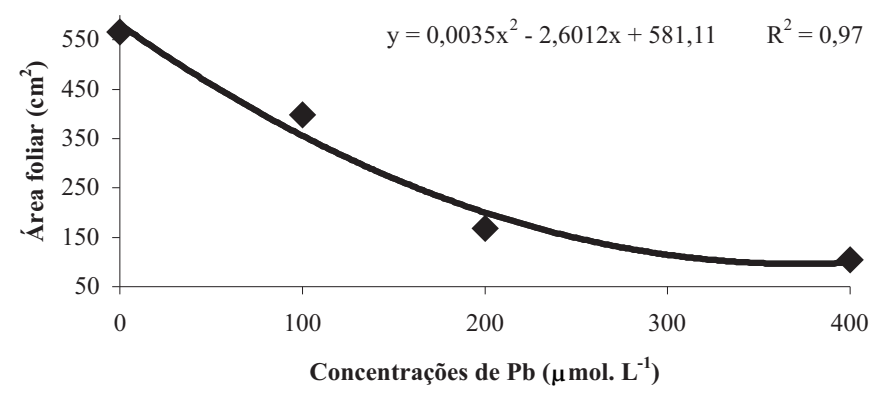

Figura 2. Áreas foliares de plantas de feijão-de-porco (Canavalia ensiformes L.) cultivadas em sistema hidropônico, submetido a diferentes concentrações de chumbo. Equações de regressão polinomial quadrática, estatisticamente significativas a $5 \%$, estimadas pelo programa SANEST.
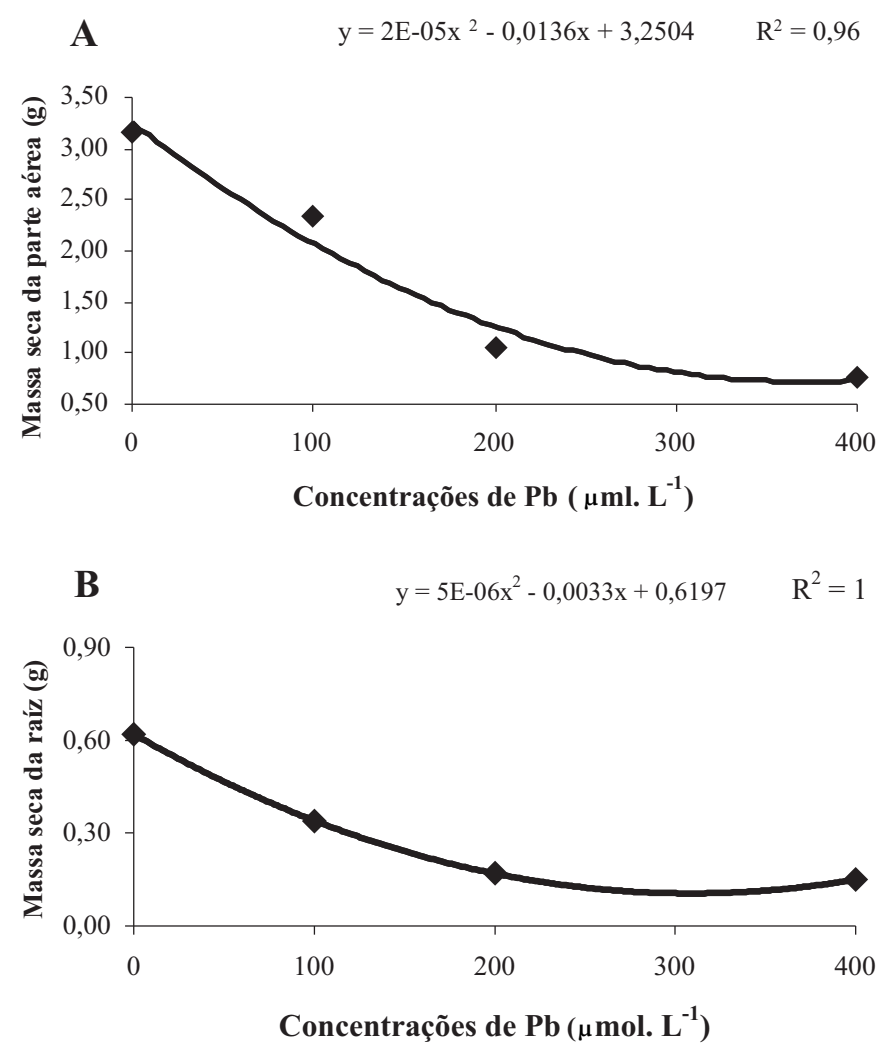

Figura 3. Médias das massas secas da parte aérea (A) e das massas secas das raízes (B), de plantas de feijãode-porco (Canavalia ensiformes L.) cultivadas em sistema hidropônico, submetido a diferentes concentrações de chumbo. Equações de regressão polinomial quadrática, estatisticamente significativas a $5 \%$, estimadas pelo programa SANEST. 

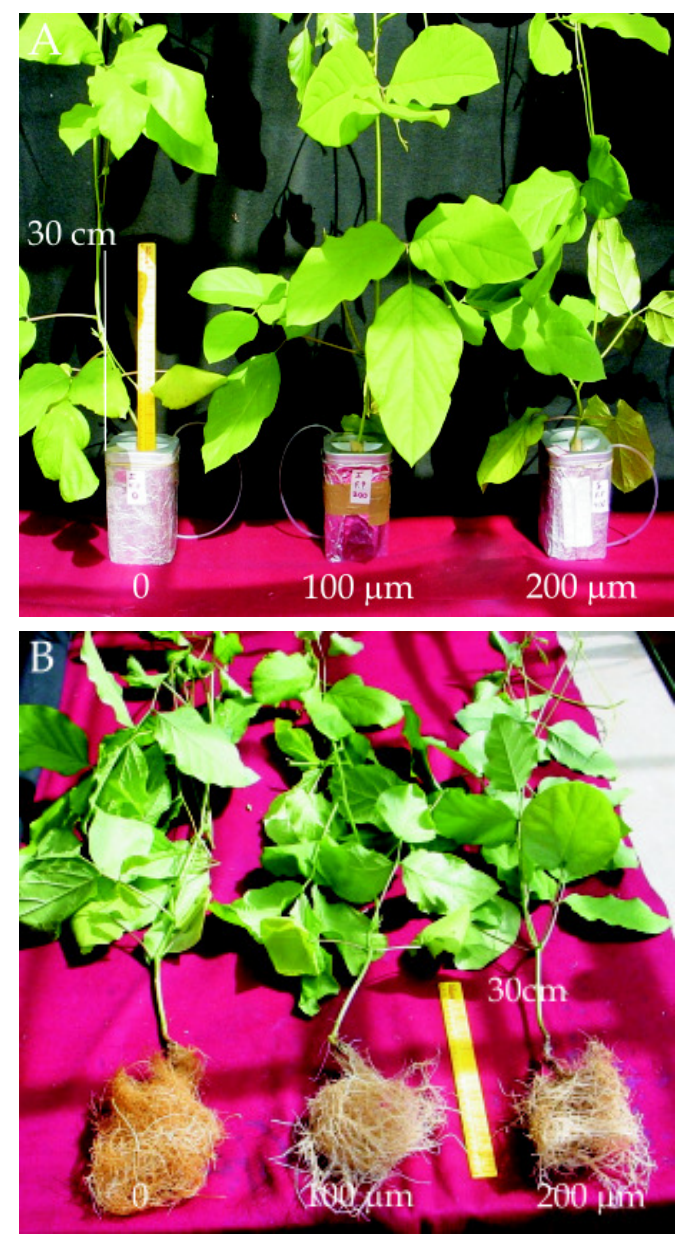

Figura 4. Aspecto das plantas de Canavalia ensiformes L. cultivadas em sistema hidropônico, submetidas a diferentes concentrações de $\mathrm{Pb}$.

Nas análises das concentrações de chumbo das soluções nutritivas em que foram desenvolvidas as espécies em sistema hidropônico, verificou-se que as plantas removeram $84 \%, 67 \%$ e $88 \%$ dos 100,200 e $400 \mu \mathrm{mol} . \mathrm{L}^{-1}$, respectivamente, do $\mathrm{Pb}$ aplicado na solução (Figura 5-A), demonstrando a eficiência dessas espécies em fitoextrair metal pesado.

Em relação ao conteúdo de $\mathrm{Pb}$ da parte aérea (Figura 5-B), verificou-se que o coeficiente de determinação foi baixo, demonstrando que com o aumento da concentração de $\mathrm{Pb}$ aplicado, não houve o respectivo aumento de conteúdo de $\mathrm{Pb}$ na parte aérea. O mesmo não ocorreu com as concentrações de $\mathrm{Pb}$ nas raízes, que foram maiores e crescentes com o aumento de $\mathrm{Pb}$ aplicado $\left(\mathrm{r}^{2}=0,98\right)$ (Figura 5-C). O alto conteúdo de $\mathrm{Pb}$ nas raízes das plantas de feijão-deporco, de acordo com as concentrações utilizadas, demonstrou alto nível de absorção e de acúmulo desse metal nesse órgão (Figura 5-C). Nas plantas de Carex rostrata, Eriophorum angustifolium e Phragmites australis, crescidas em condições hidropônicas, não houve modificações no crescimento em presença de $\mathrm{Pb}$. Como em outros casos já descritos, o $\mathrm{Pb}$ absorvido concentrou-se nas raízes (Stoltz e Greger, 2002). Em Pinus radiata, verificou-se que o $\mathrm{Pb}$ concentrado nas raízes foi observado exclusivamente nas paredes celulares desses órgãos e em Chamaecytsus palmensis na lamela média e espaços intercelulares (JARVIS, 2002).
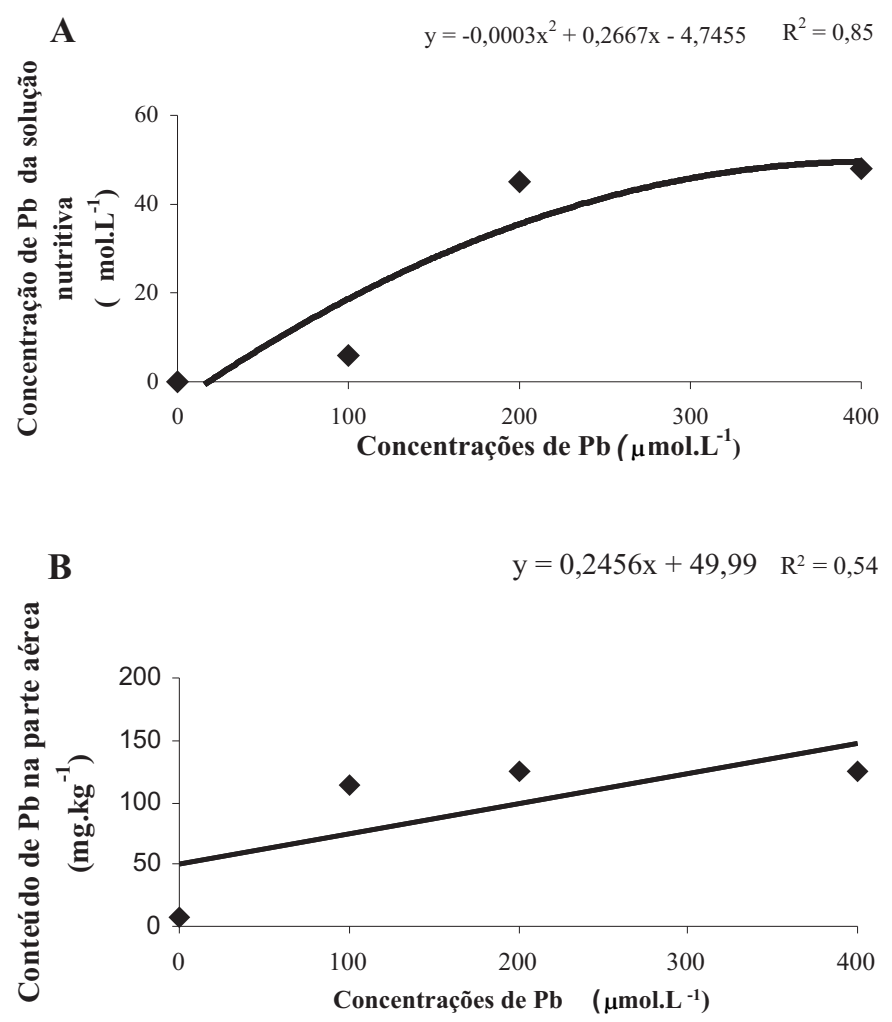

C $y=0,0003 x^{2}+0,1711 x-2,5117 \quad R^{2}=0,98$

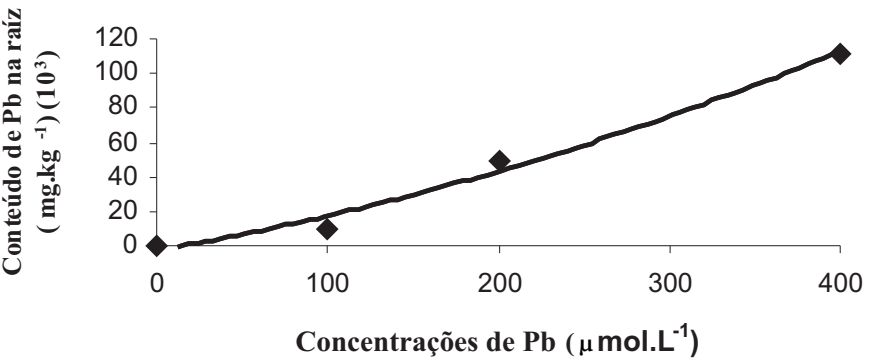

Figura 5. Médias das concentrações de chumbo na solução nutritiva (A), do conteúdo de chumbo na parte aérea (B) e do conteúdo de chumbo nas raízes (C), em que foram desenvolvidas plantas de feijão-de-porco (Canavalia ensiformes L.) em sistema hidropônico. Equações de regressão polinomial linear e quadrática, estatisticamente significativas a $5 \%$, estimadas pelo programa SANEST. 
Apesar das altas concentrações de $\mathrm{Pb}$ nas raízes, pouco foi translocado para a parte aérea de feijão-de-porco (Figura 5-B e C). Relacionando esses resultados com os dados de crescimento, pode-se inferir que as baixas concentrações de $\mathrm{Pb}$ verificadas na parte aérea não foram suficientes para provocar inibição do desenvolvimento dessa espécie, o que não ocorreu na concentração maior $\left(400 \mu \mathrm{mol}^{-L^{-1}}\right.$ de $\left.\mathrm{Pb}\right)$. Jarvis (2002), Romeiro (2005) e TSEn et al., (2002) relatam haver forte habilidade das raízes em reter quantidades significativas de $\mathrm{Pb}$, restringindo simultaneamente a translocação para a parte aérea. AldRICH et al. (2004), estudando plantas de Prosopis spp., observaram maior potencial de acumular elevados níveis de $\mathrm{Pb}$ nas raízes desta espécie, translocando-se pouco para a parte aérea. YURUK e BOzKURT (2006) também observaram acúmulo de $\mathrm{Pb}$ nas raízes de feijão. No presente trabalho, pode-se inferir que as altas concentrações de $\mathrm{Pb}$ retidas nas raízes conferiram uma tolerância dessa espécie ao $\mathrm{Pb}$.

O potencial de uma espécie vegetal em fitorremediar solos contaminados pode ser avaliado de diferentes maneiras. Conforme RASKIN et al. (1994), plantas hiperacumuladoras de $\mathrm{Pb}$ são aquelas capazes de extrair e acumular em seus tecidos valores superiores a $1.000 \mathrm{mg} \mathrm{kg}^{-1}$ de $\mathrm{Pb}$ na massa seca. Em feijão-de-porco as concentrações de $\mathrm{Pb}$ encontradas sempre excederam estes valores, variou de 10.502 a $112.455 \mathrm{mg} \mathrm{kg}^{-1}$ de $\mathrm{Pb}$ nas concentrações de 100 e 400 mmol. $L^{-1}$. Dessa forma, segundo a definição desse autor citado, pode-se inferir que as plantas de feijãode-porco, além de serem tolerantes ao $\mathrm{Pb}$, possuem características de boas hiperacumuladoras.

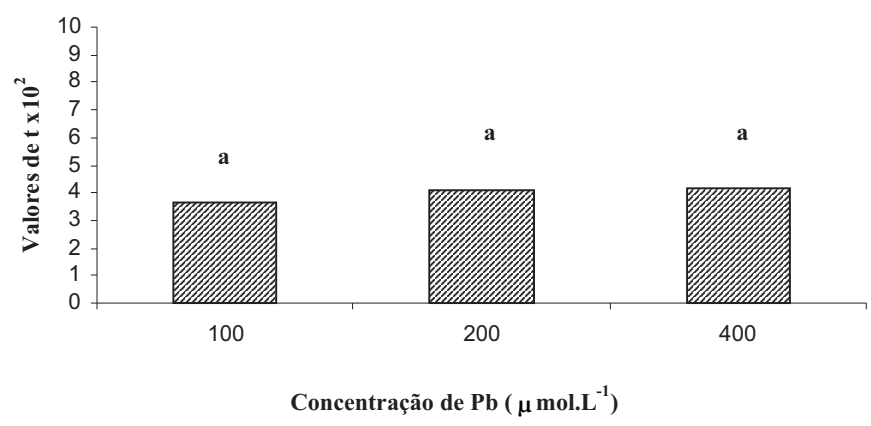

Figura 6. Médias dos fatores de transferência de $\mathrm{Pb}(\mathrm{t})$ calculadas em plantas de feijão-de-porco (Canavalia ensiformes L.) crescidas em sistema hidropônico na presença de chumbo. Letras iguais representam que não houve diferenças estatísticas significativas pelo teste Tukey a 5\%, na comparação entre as concentrações de $\mathrm{Pb}$.
Ao analisar os valores do fator de transferência (t) do feijão-de-porco, observou-se que não houve diferença significativa entre as concentrações de 100, 200 e de 400 mmol.L (Figura $^{-1}$ (Fi) 6). Segundo esse atributo, as plantas de feijão-deporco foram eficientes neste processo. Segundo Henry (2000), quanto maior for este fator, maior a absorção do contaminante e maior o potencial fitoextrator da espécie. Desta forma, pode-se inferir que o aumento das doses de $\mathrm{Pb}$ na solução não aumentou a absorção deste metal pesado pelas plantas.

Considerando-se as condições experimentais do presente trabalho, nas concentrações mais baixas de $\mathrm{Pb}$, de maneira geral, as plantas de feijão-de-porco são indicadas como potenciais hiperacumuladoras de chumbo.

\section{CONCLUSÃO}

Canavalia ensiformes L. (feijão-de-porco) é uma espécie tolerante e hiperacumuladora de $\mathrm{Pb}$, principalmente nas raízes, e por essas características deve ser investigado seu potencial fitoextrator para esse metal em condições de campo.

\section{REFERÊNCIAS}

ABREU, M.F.; ABREU, C. A.; BATAGLIA, O.C.; MARQUES, J.C. Protocolo analítico para caracterização química de substrato para plantas. Campinas: Instituto Agronômico. Centro de Pesquisa e Desenvolvimento em Solos e Recursos Ambientais, 2000. (não publicado)

ACCIOLY, A.M.A.; SIQUEIRA, J.O. Contaminação química e biorremediação do solo. Tópicos em Ciência do Solo, Viçosa, v.1, p.299-351, 2000.

ALDRICH, MV.; ELLZEY, JT.; PERALTA-VIDEA, JR.; GONZALEZ, JH.; GARDEA-TORRESDEY, JL.. Lead uptake and the effects of EDTA on lead-tissue concnetrations in the desert species mesquite (Prosopis spp.). International Journal of Phytoremediation, Texas, v.6, n.3, p.195-207, 2004.

BRASIL. Ministério da Agricultura. Regras para análise de sementes. Brasília: SNDA/ DND.V/ CLAV, 1992. 365 p.

BOONYAPOOKANA B.; PARKPLAN P.; TECHAPINYAWAT S.; DELAUNE R.D.; JUGSUJINDA A..Phytoaccumulation of lead by sunflower (Helianthus annuus), tobacco (Nicotiana tabacum), and vetiver (Vetiveria zizanioides). Part A. Toxic/ Hazardous Substances \& Environmental Engineering, Journal of Environmental Science and Health, Phatumthani, v.40, n.1, p.117-137, 2005.

CETESB. Companhia de tecnologia de saneamento ambiental. Relação de áreas contaminadas no Estado de São Paulo. Disponível em: <http://www.cetesb.sp.gov.br> Acesso em 28 de junho de 2005. 
CHANTACHON, S.; KRUATRACHUE, M.; POKETHITIYOOK P.; UPATHAM S.; TANTANASARIT S.; SOONTHORNSARATHOOL, V. Phytoextraction and accumulation of lead from contaminated soil by vetiver grass: Laboratory and simulated field study. Water Air and Soil Pollution, Dordrecht, v. 154, p.37-55, 2004.

EUN S.O.; YOUN, H.S.; LEE, Y.. Lead disturbs microtubule organization in the root meristem of Zea mays. Physiologia Plantarum, Copenhagen, v. 110, p.357-365, 2000.

FURLANI, A. M. C.; FURLANI, P. R.. Composição e pH de soluções nutritivas para estudos fisiológicos e seleção de plantas em condições nutricionais adversas. Campinas: Instituto Agronômico, 1988. 34p. (Boletim Técnico, 121)

GARBISU, C.; ALKORTA, I.. Phytoextraction: a cost effective plant-based technology for the removal of metals from the environment. Bioresource Technology, Essex, v.77, p. 229-236, 2001.

GRATÃO, P.L.; PRASAD, M. N.V.; CARDOSO, P. F.; LEAD, P.J.; AZEVEDO, R.A.A.. Phytoremediation: green technology for the clean up of toxic metals in the environment. Brazilian Journal of Plant Physiology, Campinas, v.17, n.1, p.53-64, 2005.

HENRY, J.R..An overview of the phytoremediation of lead and mercury. National Network of Environmental Management Studies (NNEMS). Washington, D. C.: U.S. Environmental Protection Agency, 2000. p.51.

HORNG, T.; SU-CHIACHUN; KING-VAE.; TSEN-JH; SU-CC. Heavy metals absorption of hydroponic Chinese kale. Journal of Agriculture and Forestry, Taipei, v.51, n.1, p. 73-83, 2002.

JARVIS, M.D.; LEUNG, D.W.M. Chelated lead transport in Chamaecytisus proliferus (L.f.) link ssp. proliferus var. palmensis (H. Christ): an ultrastructural study. Plant Science, Amsterdam, v.161, n.3, p. 433-441, 2001.

JARVIS, M.D.; LEUNG, D.W.M..Chelated lead transport in Pinus radiata: an ultrastructural study. Environmental and Experimental Botany, Oxford, v.48, n.1, p. 21-32, 2002.

JONES, L.H.P.; CLEMENT C.R.; HOPPER, M.J. Lead uptake from solution by perennial ryegrass and its transport from roots to shoots. Plant Soil, Dordrecht, v. 38, p.403414, 1973.

KHAN, A.G.; KUEK, C.; CHAUDHRY, T.M.; KHOO, C.S.; HAYES, N.J. Role of plants, mycorrhizae and phytochelators in heavy metal contaminated land remediation. Chemosphere, Oxford, v.41, p.197-207, 2000.

KOSOBRUKHOV, A.; KNYAZEVA, I.; MUDRIK, V. Plantago major plants responses to increase content of lead in soil: growth and photosynthesis. Plant Growth Regulation, Dordrecht, v.42, p.145-151, 2004.

LANE S.D.; MARTIN E.S. A histochemical investigation of lead uptake in Raphanus sativus. New Phytologist, London, v.79, p.281-286, 1977.
LARBI, A.; MORALES, F.; ABADIA, A.; GOGORCENA,Y.; LUCENA, J.J.; ABADIA, J. Effects of $\mathrm{Cd}$ and $\mathrm{Pb}$ in sugar beet plants grown in nutrient solution: induced Fe deficiency and growth inhibition. Functional Plant Biology, Victoria, v.29, n.12, p.1453-1464, 2002.

LASAT, M. M. Phytoextraction of toxic metals: A review of biological mechanisms. Journal of Environmental Quality, Madison, v.31, p.109-120, 2002.

LUBBEN, S; SAUERBECK, D The uptake and distribution of heavy-metals by spring wheat. Water Air dnd Soil Pollution, Dordrecht, v.57, n.8, p.239-247, 1991.

MALAVOLTA, E. Fertilizantes e seu impacto ambiental: micronutrientes e metais pesados: mitos, mistificação e fatos. São Paulo: Petroquímica, 1994.153p.

MARQUES, T.C.L.S.M.; MOREIRA, A.M.S.; SIQUEIRA, J.O. Crescimento e teor de metais de mudas de espécies arbóreas cultivadas em solo contaminado com metais pesados. Pesquisa Agropecuária Brasileira, Brasília, v.35, n.1, p.121-132, 2000.

MICHALSKA, G. Crossing effects of Belgian Landrace boars with Polish Large White, Hampshire and Duroc sows. Pollution Journal of Food Nutrition Science, Bruxeles, v.10, n.51, 139141, 2001 .

QU, RL.; LI, D.; DU, R.; QU, R. Lead uptake by roots of four turfgrass species in hydroponic cultures. Hortscience, Alexandria, v.38, n.4, p. 623-626, 2003.

RAMANI; S.; SHAIKH, M.S.; SUSEELAN, K.N.; KUMAR, S.C.; JOSHUA, D.C.; RAMANI, S. Tolerance of Sesbania species to heavy metals. Indian Journal of Plant Physiology, New Delhi, v.7, n.2, p.174 - 178, 2002.

RASKIN, I., KUMAR, PBA. N., DUSHENKOV, J.R.; SALT, D.E. Bioconcentration of heavy metals by plants. Current Opinions Biotechnology, Philadelphia, v.5, p.285 - 290, 1994.

ROMEIRO, S. Potencial de Ricinus communis L. Helianthus annus L. e Canavalia ensiformes L. como extratoras de chumbo em solução nutritiva. 2005, 84f. Dissertação (Mestrado em Agricultura Tropical e Subtropical) - Instituto Agronômico, Campinas, 2005.

SHARMA, P.; DUBEY, R.S. Toxic Metals in :Lead toxicity in plants. Brazilian Journal Plant Physiology, Taipei, v.17, n.1, p.35-52, 2005.

SHEN, Z. G., LI, X. D., WANG, C. C., CHEN, H. M.; CHUA, H. Lead phytoextraction from contaminated soil with highbiomass plant species. Journal Environmental Quality, Madison, v.31, p.1893-1900, 2002.

STOLTZ, E.; GREGER, M. 2002. Accumulation properties of $\mathrm{As}, \mathrm{Cd}, \mathrm{Cu}, \mathrm{Pb}$ and $\mathrm{Zn}$ by four wetland plant species growing on submerged mine tailings. Environmental and Experimental Botany, Paris, v.47, n.3, p.271-280, 2002.

TANG, S.; WILKE, B.; BROOKS, R.; TANG, S. Heavy metal uptake by metal tolerant Elsholtzia haichowensis and Commelina communis from China. Communications in Soil Science and Plant Analysis, New York, v.32, p.895-905, 2001. 
TSEN, JENGHORNG, SU, CHIACHUN; KING, VAE, TSEN, JH; SU, CC. Absorption of various heavy metals by hydroponic water spinach. Journal of Agriculture and Forestry, Taipei, v.50, n.4, p.1-11, 2002.

VERMA S.; DUBEY, R.S. Lead toxicity induces lipid peroxidation and alters the activities of antioxidant enzymes in growing rice plants. Plant Science, Amsterdam, v.164, p.645655, 2003.
WIERZBICKA, M.; ANTOSIEWICZ, D. How lead can easily enter the food chain - a study of plant roots. Science Total Environmental Supplement, Washington, v.1, p.423 - 429, 1993.

YURUK, A.; BOZKURT, M. A. Heavy metal accumulation in different organs of plants grown under high sewage sludge doses. Fresenius Environmental Bulletin, Berlin, v.15, n.2, p.107 - 112, 2006. . 